\title{
Micro level architecture for Automatic Cleaning Device
}

\author{
Ayushi Nigam, Dr. Hari Om Sharan
}

\begin{abstract}
According to the present scenario, India is one of the most recently growing and developing country. The internet is continuously changing and evolving field of technology. The main communication form of present Internet is human to human. The Internet of Things (IoT) can be considered as the future evaluation of the Internet that realizes machine-to-machine (M2M) learning. Thus, IoT provides connectivity for everyone and everything. The IoT embeds some intelligence in Internet connected objects to communicate, exchange information, take decisions, invoke actions and provide amazing services. IoT is one of the evolving area of technological world. IoT Technology is becoming popular day by day because of its acceptance in various areas for solving much of real life problems like as know water is one of our precious resource and for life on earth without water life is not possible so for measuring its quality an IoT based water quality system has developed, similarly In medical field an IoT based patient health monitoring system has developed, In field of environmental development IoT based waste management system has developed, Education sectors are providing smart classrooms which provide an interactive environment for teaching, again contributing towards IoT and also Industrial sector adopting the various means for controlling their processes through an internet. An IoT based devices not only reduces the use of man power but also less time consumable and energy efficient. This research paper focuses on the development of very useful IoT based micro level architectural device that resolves the very important real life problem of cleaning in various fields like household, shopping malls, industries, banking sector etc.
\end{abstract}

Index Terms - Internet of things(IoT) Technology, micro level architectural device

\section{INTRODUCTION}

According to today's scenario every person is running in the race of chasing the evolving technology, due to this the person don't have sufficient amount of time to do his personal works which are the part of our daily life, one of them is house cleaning so for that, this paper focuses on the development of an IoT based micro level architecture to deal with the problem of house cleaning. The automatic cleaning device that is developed under this paper performs the cleaning of the particular enclosed area by using the concept of blind cleaning i.e. the automatic cleaning device performs motion on the basis of predefined statistics receiving to the device from any remote location with the help of cloud computing technology to collect the unclean dirty patches by moving with some constant speed in whole area by assuming that completely enclosed area is full of dirty patches. As we know technology is the perfect blend of innovation and ideation that

Ayushi Nigam, M.Tech. Scholar, CSE Department, FET, RAMA University, KANPUR

Dr. Hari Om Sharan, Professor, CSE Department, FET, RAMA University, KANPUR conceptualizes to form a platform, which is suitable for operating various IoT device developments taking place. This IoT device reduces the manual work because in this model an automation feature is inbuilt due to which this device does not require any human intervention to perform its working.

This micro level architecture does not conceptualizes the Traditional IoT architectural approach, which mainly used for designing of large scale IoT devices, the micro level architecture for automatic cleaning device follows the redesigned three layered architectural approach .

\section{RELATED WORK}

Several countries like Japan, Germany and America are trying to develop cleaning robots since 1991. Companies like GeckoSystems (US) and Probotics (US) launched two automated floor cleaning systems before year 2000. By year 2000 and 2001 companies like Electrolux (Sweden), K" archer (Germany), and Dyson (UK) launched more commercial versions of automated floor cleaners. Whenever any company builds a system the success of that system is based on two very important quality attributes namely performance and cost. Systems designed by above-mentioned companies were having somewhat good performance but they were too expensive for household use. Due to which their products were only purchased by industries. In order to develop any architectural device not only fulfillment of functional requirement is important but also the establishment of tradeoff among the quality attributes.

These companies also targeted household vacuum cleaners and floor cleaning machines. These household vacuum cleaners and floor cleaning machines was suffering from two major problems namely performance and durability. Household versions like Robot Vacuum Cleaner developed by Electrolux in Sweden in 1997 [1], Robo Cleaner developed by K"archer in Germany in 1999 [1], DC06 developed by Dyson in UK in 1999 [1], Cye developed by Probotics Inc. in US in 1999 [1], CareBotTM PCR developed by Gecko Systems in US in 1998 [1] are having

Low performance and Low durability. Due to these two basic problems they are not in household use anymore. Many other companies are struggling hard till date in order to attain good performance and durability.

Cost of cleaning is also an issue with respect to quality of cleaning. Many household wives do not want to compromise with quality of cleaning. Some way or the other, this equipment's are not satisfying the need of the common housewife. Apart from this, other systems which are using very complex technologies like radar motion planning, infrared based object detection, and so on, are very costly. The path planning algorithms and cleaning algorithms are integrated together on a same piece of hardware. So the overall performance of system becomes slow and degraded. 
Fig. 1, 2, 3, 4 are basically taken from reference 1 . They provide some visual representations of several automatic vacuum cleaners used in world

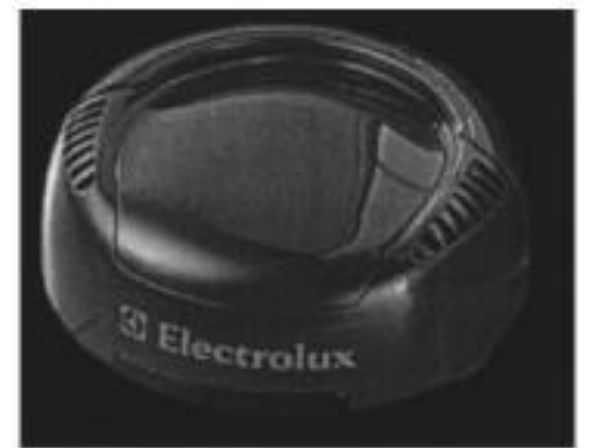

Fig. 1:- Showing Electrolux Vacuum Cleaner [1]

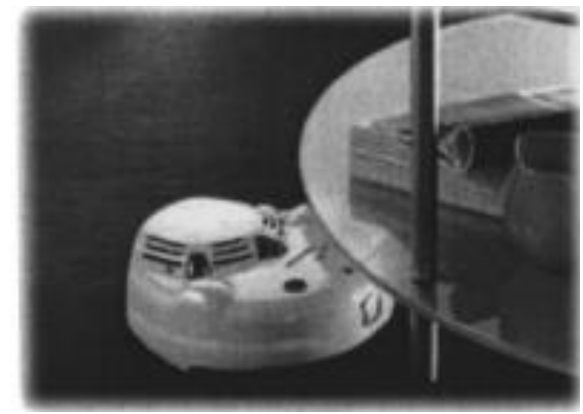

Fig. 2:-. Showing Karche Vacuum Cleaner [1]

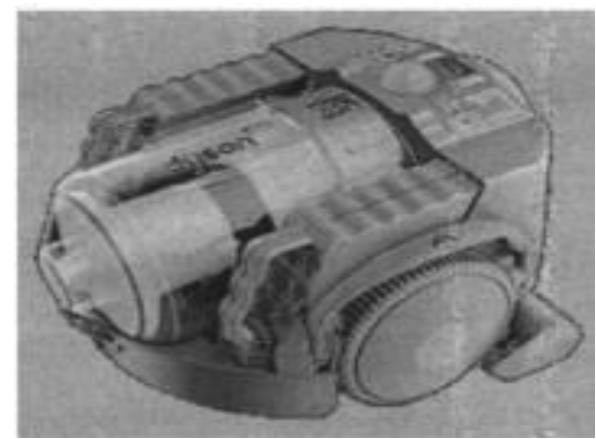

Fig. 3. Showing DC06,Dyson Vacuum Cleaner [1]

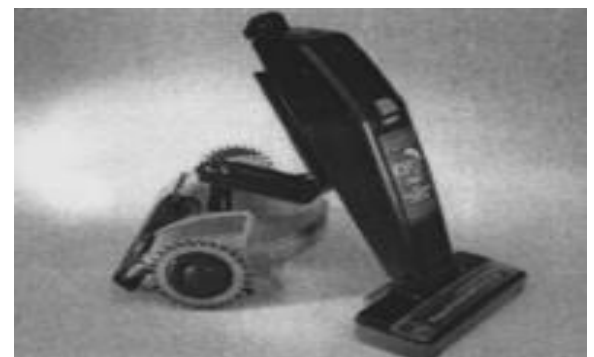

Fig. 4:- Showing Cye, Probotic Vacuum Cleaner [1]

\section{TRADITIONAL APPROACH}

In recent time we are having a very generic and bulky architecture for IOT devices. These architecture are based on canonical 4 layer architecture which consist of Device layer, Network layer, Application layer and above all the business layer. The traditional approach for designing IOT devices or embedded systems is sufficient for designing the macro architectures which requires costing of almost thousands of rupees and it also requires complete network protocol stack but this architecture is not appropriate for designing small scale systems because for such type of systems do not requires complete protocol stack and in traditional layered architecture the network layer (edge IT phase) integrated with heavy protocols like HTTP due to which it suffers from latency problem. To avoid all these things the micro level architecture eliminates unwanted elements from network layer so that complexity of the network layer gets reduced.

This Traditional system consists of 4 stages of IOT architecture. In essence, IOT architecture is the system of numerous elements: sensors, protocols, actuators, cloud services and layers. Such a number is chosen to steadily include these various types of components into a sophisticated and unified network. In this architecture layers are distinguished in order to track the consistency of the system before starting the process of each layer.

BUSINESS LOGIC LAYER
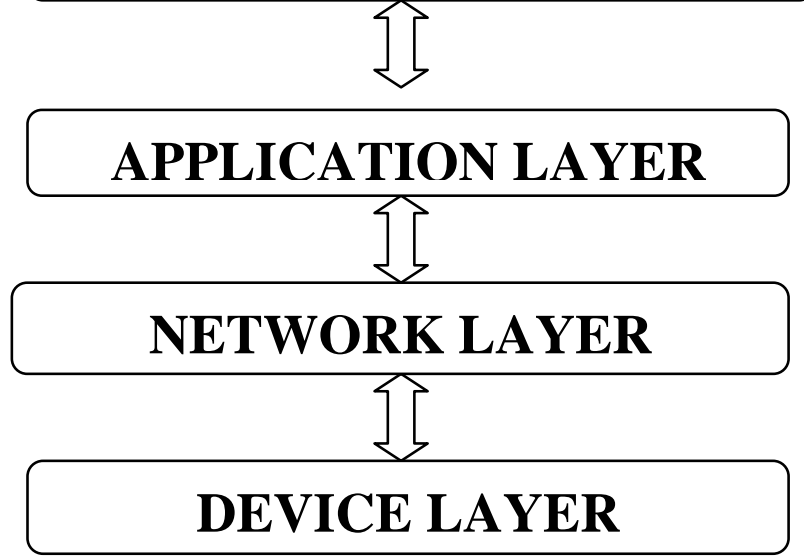

Fig 5:- Traditional 4 Layered IOT Architecture

\section{A. DEVICE LAYER: -}

This stage is the initial stage of traditional IOT architecture includes all components or devices, machines, people tool etc which are communicating with each other. This layer also consists of networked things wireless sensors and actuators. A better term for a sensor is a transducer. A transducer is any physical device that converts one form of energy into another. So, in the case of a sensor, the transducer converts some physical phenomenon into an electrical impulse that can then be interpreted to determine a reading. The sensors are the source of IOT data and having an ability firstly to sense the outer world for eg:- Turn off the light, turn on the air conditioner, adjust the refrigerator temperature etc convert the information about the outer world into data for analysis where as there are another type of transducers are called actuators which actually works in reverse direction of sensors. It takes electrical inputs and convert it into the physical motion. In a typical IoT system, a sensor may collect information and route to a control centre where a decision is made and a corresponding command is sent back to an actuator in response to that sensed input.

\section{B. NETWORK LAYER: -}

This layer plays a very important role in transmitting the data sensed by the sensor device into the network. In this layer firstly sensor data aggregation system transfer the data after 
converting data in digital form into analog form and vice versa. Internet getaways and data acquisition systems (DAS) both appear here. The vital importance of this stage is to process the enormous amount of information collected on the previous stage and squeeze it to the optimal size. Internet gateways work through Wi-Fi wired LANs and performs further processing. In short, Stage 2 makes data both digitalized and aggregated.

\section{APPLICATION LAYER:-}

This layer interacts with user directly and this layer performs action on the basis of signals received from network layer or transferred the user generated response to device layer in order to perform the action through the device

This layer consist of edge IT system in order to perform enhanced analytics and pre processing here. On the basis of action performed in this layer data is transferred to the data centre for storage. This layer also consists of machine learning and visualization technologies. The machine learning algorithm are implemented in Cloud which receives data on the basis on Wi-Fi module from any remote location and generate the appropriate response and transfer the response to device layer for devices to perform an action and also transfers the response to business logic layer for maintaining the updated status of device.

\section{BUSINESS LOGIC LAYER:-}

This is last layer of IOT architecture. This layer includes the graphs, bar charts, pie charts for representing the status of the devices or components interacting with each other. On the basis of the response of this layer the data get recorded into storage. This layer refers to initiating a user's control over the structure - if only your result doesn't include full automation, of course. The main tasks here are visualization and management. After this stage the overall system turns into a circle where user now perform the repeated action and sends the commands to sensors and actuators in stage 1 to perform some actions.

Fig 6:- Traditional 4 Layered IOT System

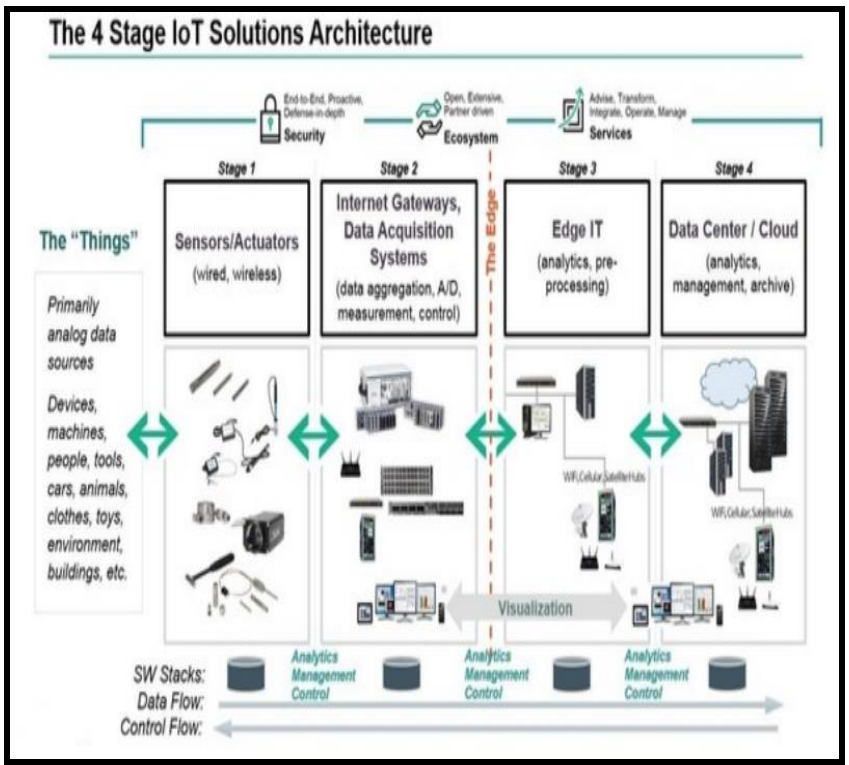

3.1 Pros and Cons of Traditional 4 Layered IOT Architecture

\section{1) Pros of Traditional Approach}

a) Traditional approach is perfect for macro devices because for such devices the network layer requires to integrate with heavy protocols and also require the complete protocol stack in order to transmit and receive the data between device layer and application layer.

b) Delivering the quality attributes also by fulfilling the functional requirements is really herculean task and establishes the tradeoffs between the two is not an easy task

c) The traditional architecture is a 4 layered architecture. Each of which layer performs a specific functionality but the main problem with this architecture is the latency problem which arises when this approach make a use of Cloud Computing technology.

\section{2) Cons of Traditional Approach}

a) Traditional approach requires the costing of thousands of rupees but this approach works perfectly for large scale devices.

b) The solution for the problem of Cloud based IOT architecture is Fog Computing.

\section{THREE LAYERED APPROACH}

In three layered architecture the device layer is merged with network layer, there is one new layer is get introduced i.e. logic layer, this layer is somewhat overlapped with Device layer (DL)and in this architecture the business logic layer (BLL) is integrated with application layer which is called as business logic layer The detailed description of each layer given below-

a) Device Layer: - This layer consists of electronic devices or components or circuits inside the devices include logic gates, combination of these circuits forms a big circuit. The network layer is sub merged with device layer because in case of small scale systems this architecture does not requires the network layer to integrate with heavy protocols because for small scale systems the architecture does not require complete protocol stack.

b) Logic Layer: - This layer includes logic codes or operation codes. This code controls the functioning of logic circuits lie inside the devices. The operation codes act as a mediator between device layer and business logic layer. This layer receives the signal through the $\mathrm{Wi}-\mathrm{Fi}$ module from the device which is located at any remote location then on the basis of this signal with the help of operation code the particular response generated; on the basis of this response the device performs an action.

c) Business Logic Layer:- The device that is responsible of updating the status of devices that are the part of device layer comes under business logic stage. In three layer architecture the application layer is merged with business logic layer and called as business logic layer. This layer not only interacts with Server also interacts with user who generate the response then this response is get transmitted to the microcontroller then micro controller perform function on the basis of operation code fed into micro controller then on the basis of signal from micro controller the electronic device performs an operation. This layer not only updates the response of device but also updates its status on the database running on the server located at any remote location. 
Fig 7:- Three Layered Approach

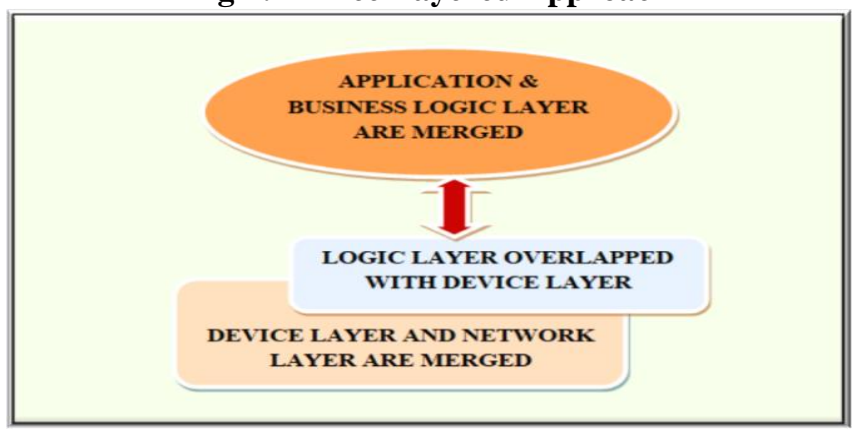

Fig 7:- Detailed Schematic Representation of Three Layered Approach

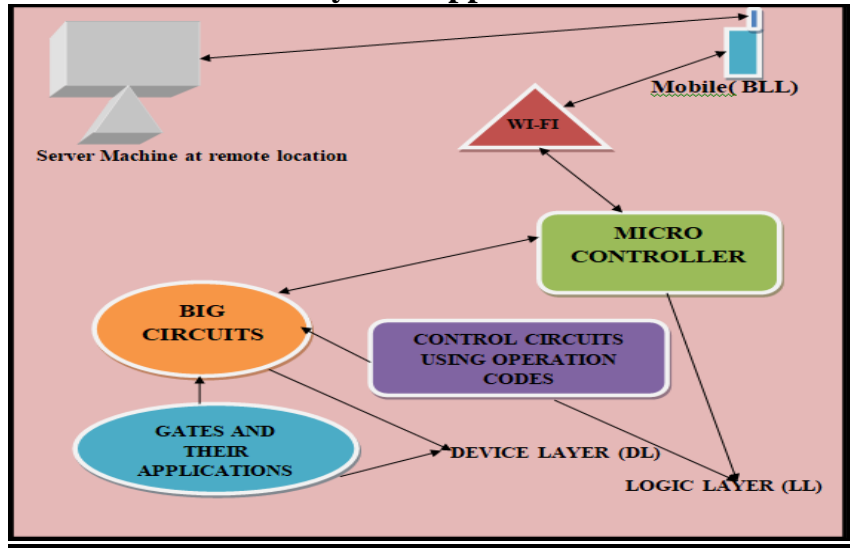

\section{DESIGN OF MICRO LEVEL ARCHITECTURE FOR AUTOMATIC} Cleaning DeVICE

The designing of micro level architecture for automatic vacuum cleaner is based on three layered architecture. This layered architecture consist of network layer merged with device layer, overlapping logic layer and also the application layer merged with business logic layer. The device layer consist of logic devices like L293D motor driver IC, Vacuum Cleaner where as logic layer consist of microcontroller device i.e. arduino and the business logic layer consist of NodeMCU i.e. Wi-Fi module.

In present day scenario designing a new system with respect to a given architecture is easy, but to design architecture is really a tough job because by designing any architecture we have to deal with quality attributes. For e.g.:If we a system secure then it is very much possible that system response will be slow, so security and response time are two quality attributes if one increases then other decreases.

So there are several qualities attributes which we have to think and take care of while designing a particular system and this quality attributes are totally dependent on the architecture, any project must be classified into two parts -

Functional Requirement: - The functional requirement means that develop the working capability inside the system. Operation codes are used to make the device functional. This operation code gets fed into the logic devices then on the basis of received signal the logic devices performs action.

Quality Attributes :- The quality attributes are modularity, scalability, development time, overall performance establish the tradeoffs among all leads to development of an architecture which is good and fruitful for designing small scale systems, which consist of reduced development time and the quality of the systems are improved and also the cost decreases drastically.
Fig 7:- Design of Micro Level Architecture for Automatic Cleaning Device

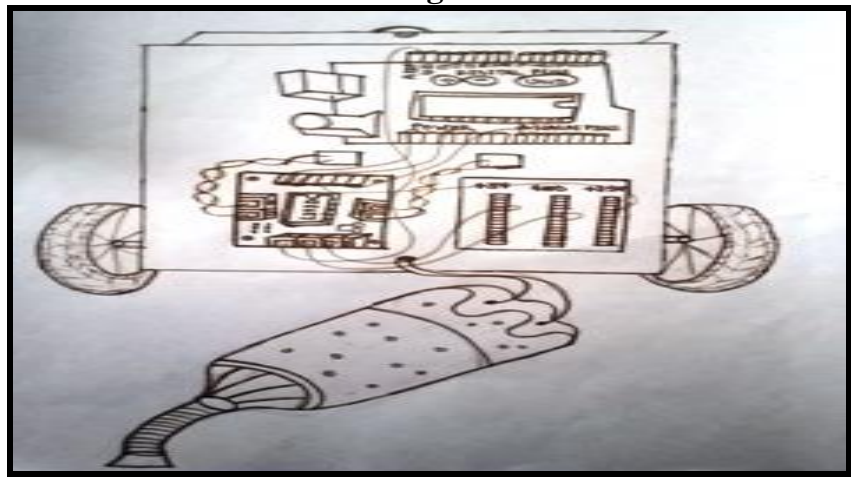

VI. FUNCTIONS PERFORMED BY MICRO LEVEL ARCHITECTURE FOR AUTOMATIC CLEANING DEVICE

The functions performed by hardware model is described below-

\section{A. Motion Planning: -}

The motion planning function is performed by user who interacts with business logic layer. The user decides the motion (Left Turn or Right Turn or Forward or Backward) with the help of PC or mobile device via Wi-Fi module. On the basis of signal from user the NodeMCU transmit the signal to arduino then motor driver IC is connected with arduino then on the basis of data received from arduino motor driver IC directs the cleaning device and perform that particular motion.

\section{B. Distance Diagnostics: -}

The distance diagnostics is basically the analysis of distance, how much steps that the device have to move to reach to the dirty patch and to clean it. The distance diagnostics feature in our architecture is implemented in local server but in actual it is implemented in cloud but now in my thesis local server is treating as cloud.

The distance diagnostics is performed by machine learning algorithm that is executing in our local server, this algorithm decides the appropriate distance that the device must be covered in order to clean the dirty patch.

\section{Data Storage:-}

The data storage step deals with business logic layer for storing the data on the server via Wi-Fi module. Then on the basis of data stored on the server the BLL updates the status of the device through the Pie graphs, bar charts etc

\section{Feature Selection:}

The feature selection step deals with deciding the features like (motion planning, distance diagnostics, data storage). Business logic layer deals with feature selection step through the Wi-Fi module

The machine learning algorithm that is used here for implementing Local Server as Cloud is " $\mathbf{K}$ Means Clustering".

\section{Working of AUtomatic Cleaning DeVICE}

The conceptual working of automatic vacuum cleaner is shown in above fig 5.4. The three layered architecture consist of three layers Device layer, Logic layer, and also the business logic layer. Here this thesis focusing on the designing of 
vacuum Cleaner that blindly moves over the complete room the Collect the dirt patches from all over the room clean the complete room and returned back to its original position. Here the local Server is serving as a cloud where machine learning algorithm is implemented which provides the appropriate motion for the micro level architecture. The mapping of over all working of automatic vacuum cleaner in terms of three layered architecture is given below-

a) Device Layer: - This architecture consists of cleaning device, L293D motor driver both are the part of this layer. These devices or a logic components perform functioning on the basis of the signal received from the business logic layer via logic layer. The network layer in three layered architecture is merged with this layer. Because the network layer for small scale systems does not requires to integrate with heavy protocols and also does not requires the complete protocol stack For designing the small scale devices the server that we uses is the local server so in order to send and receive data from local server does not requires the network layer to face the latency problem.

b) Logic Layer: - The devices that are the part of logic layer are a micro controller device i.e. arduino. This micro controller device works on the basis of operation code that is fed into arduino. Here arduino is connected with the motor driver and the motor driver perform working on the basis of signal transferred by arduino, received via $\mathrm{Wi}-\mathrm{Fi}$ module through mobile device or through the personal computer.

c) Business Logic Layer: - In three layered architecture the business logic layer is merged with application layer So in this architecture the user directly interacts with business logic layer. Mobile device or the PC are the part of business logic layer, whatever the signal that user generates then this signal is get transferred to the local server through the Wi-Fi module i.e. NodeMCU where the machine learning algorithm is running then this algorithm generates makes the planning about the motion of the cleaning device then the data from the server is transferred to the arduino device through the $\mathrm{Wi}-\mathrm{Fi}$ module then the signal from arduino is get transferred to the motor driver then in that way the cleaning device perform the cleaning and after that cleaning device returned back to its original position.

Fig 8:- Conceptual Working of Automatic Cleaning Device

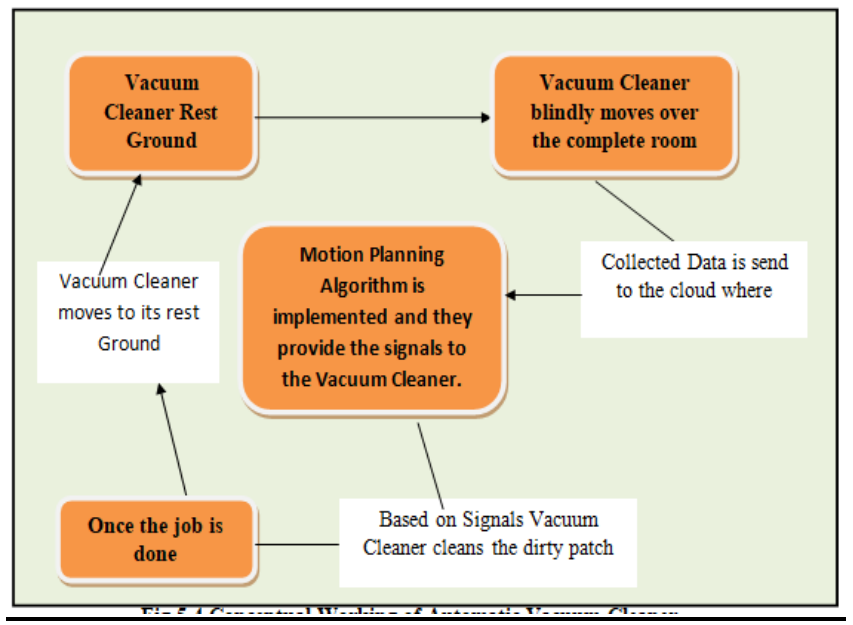

This architecture not only resolves one of the real life problems of house cleaning but also able to establish the tradeoffs among all of the Quality attributes. In this way this architecture performs cleaning at very low cost and also having the good overall performance by following the concept of three layered approach. This automotive feature of this device reduces the unnecessary human intervention, which also helps in reducing the time consumption.

\section{CONCLUSION}

With the help of three layered architectural approach, it becomes possible to develop the IOT device at small scale such architectural devices are not only cost effective but also having a good overall performance, presently this system is efficient to perform cleaning blindly in an enclosed area by receiving the signals from any remote location by using the concept of machine learning which is implemented in cloud without any human intervention. In future we are planning to make a use of cameras and sensors in order to detect the unclean dirty patch which not only reduces the time but also make this device energy efficient. This architecture also acts as a platform for developing all other similar kinds of devices having same architecture like the field ploughing machine, the spray gun for painting the wall etc.

\section{ACKNOWLEDGEMENT}

First of all I would like to say that God does everything for us and never takes the credit.

I would like to acknowledge Dr. Hari Om Sharan, HOD, of Computer Science and Engineering, Rama University, Kanpur for helping me out during the research phase and I really thankful to sir for that.

\section{REFERENCES}

[1]Erwin Prassler, Arno Ritter and Christoph Schaeffer and Paolo Fiorini, "A Short History of Cleaning Robots",Autonomous Robots 9, 211-226, 2000 2000 Kluwer Academic Publishers. Manufactured in The Netherlands.

[2] Siddarth Srivastava, Ayushi Nigam, Monika Arya and Shalini Lamba "Swaksh: An automatic vacuum cleaner" 25 Oct 2018

[3]Paul Stokes, "4 Stages of an IoT architecture explained in simple words" 5 Dec 2018

[4]Rafiullah Khan, Sarmad Ullah Khan, Rifaqat Zaheer and Shahid Khan “ Future Internet Internet of Things Architecture, Possible Applications and Key Challenges" 9 Dec 2012 IEEE Conference

[5] Youngkak Ma, seungwoo Kim, Dongik Oh and Youngwan Cho. "A study on development of home mess- cleanup robot McBot.” In IEEE/ASME 